\title{
Study on Hydrogen Sulfide Removal Technology in Petroleum Processing
}

\author{
Yinghua Wu \\ Chongqing Energy College \\ (Chongqing ,China 400041)
}

Keywords: Petroleum processing, Hydrogen sulfide, Removal technology

\begin{abstract}
With the rapid development of the national economy, our country puts forward higher requirements for environmental protection. Due to the presence of hydrogen sulfide in petroleum processing raw materials, and it seriously affects the zero emission requirements. This paper discusses the hydrogen sulfide removal technology and elaborated the wet oxidation method and dry oxidation method which means two kinds of H2S oxidation method, and each of them in the process of removing hydrogen sulfide in the principle, advantages and disadvantages are summarized, and this paper provides theoretical basis for the removal of hydrogen sulfide in petroleum processing.
\end{abstract}

\section{Preface}

In the current air pollution sources, industrial emissions become one of the main sources of emissions. As a result of emissions with toxic and harmful, it will cause some harm to the social ecological environment and human health. At present, with the rapid development of China's economy, in the treatment of toxic and harmful gas sources, it increased a number of scientific and technological researches and the relevant laws has been established. Although the results have been achieved, but in the face of complex industrial pollutants, it still can not meet the requirements of air pollution control. In many industrial pollutants of which $\mathrm{H} 2 \mathrm{~S}$ is one of major pollutants, it not only caused the corrosion of chemical equipments, but it will lead to catalyst poisoning and deactivation, thereby reducing the quality of products and reducing the economic efficiency of enterprises. So it is necessary to deal with the $\mathrm{H} 2 \mathrm{~S}$ in the exhaust gas to avoid more serious environmental problems for the human survival and development with a serious threat[1,2]. Therefore, the study of more efficient H2S removal technology has become the focus of today's society.

\section{Introduction of Hydrogen Sulfide Removal Technology}

In the process of petroleum refining, sulfur has great harm to production equipment in chemical equipment, and it will cause serious corrosion of equipment, so the need for timely removal to ensure the production device can carry out "safety, wide, long, full and excellent" production. In addition, the quality of the product is also affected by sulfur, in order to ensure the effectiveness of enterprises, the world's countries need to strengthen the control of sulfur content in oil control indicators. Through the control of sulfur content in petroleum processing, environmental protection and clean production can be realized, so as to ensure the quality of chemical equipment and oil in the process of refining and the environmental protection index of enterprises. For each refinery and natural gas chemical plant and other enterprises, the acid gas produced with $\mathrm{H} 2 \mathrm{~S}$ which must carry out the subsequent processing in the sulfur recovery unit, and the H2S gas is useful into sulfur residue gas by incineration to exhaust zero emissions to achieve the purpose of protecting the environment. Access to information, at present, the oxidative removal technology of $\mathrm{H} 2 \mathrm{~S}$ includes the following aspects: (1) removing hydrogen sulfide oxidation; (2) imbibing removal method;(3) adsorption removal;

(4) biological treatment; (5) removing ozone oxidation;(6) the electrochemical removal method. 
In recent years, a new type $\mathrm{H} 2 \mathrm{~S}$ removal process, biological removal process has been developed, which is suitable for the treatment of $\mathrm{H} 2 \mathrm{~S}$ tail gas with large volume and low density. Compared with the traditional H2S removal method, the biological removal method has the following advantages: (1) the equipment is simple; (2) the operation cost is low; (3) it is difficult to form the pollution. In addition, the biological removal method has a higher economic benefit when dealing with biodegradable and low concentration of gaseous pollutants. In addition, the electrochemical method has the advantages of simple operation, high efficiency, good environmental compatibility and easy automation, and there are no by-products, clean production, environmental friendly technology, and removal is very promising in the future, and it can be said that the two novel H2S removal technology will be the future direction of development.

\section{Hydrogen sulfide oxidation removal method}

At present, according to the principle of weak acid and enhanced reductive removal, the oxidation and removal of $\mathrm{H} 2 \mathrm{~S}$ mainly includes two kinds of $\mathrm{H} 2 \mathrm{~S}$ oxidation methods: wet oxidation and dry oxidation.

Dry oxidation method. The results show that H2S can be oxidized into sulfur oxide or sulfur by dry oxidation. It mainly includes Klaus method, iron oxide desulfurization method and composite zinc desulfurization.

Claus method. When the $\mathrm{H} 2 \mathrm{~S}$ is removed by Claus, the principle is that the partial $\mathrm{H} 2 \mathrm{~S}$ in the combustion furnace reacts with oxygen to form $\mathrm{S} 2 \mathrm{O}$, and then with the remaining $\mathrm{H} 2 \mathrm{~S}$ gas to produce a by-product of sulfur recovery. In order to ensure that $\mathrm{H} 2 \mathrm{~S}$ combustion can provide enough heat energy, the concentration of $\mathrm{H} 2 \mathrm{~S}$ needs to be greater than $15 \% \sim 20 \%$ to maintain a certain temperature, and to ensure the smooth reaction. This is an early discovery of $\mathrm{H} 2 \mathrm{~S}$ removal method, in 1883, it was achieved by the invention of C.F.ClauS . Because of the advantages of this method, it widely used in petroleum chemical industry, coal chemical industry, natural gas processing and other fields, the recovery of $\mathrm{H} 2 \mathrm{~S}$ gas in the exhaust, and exhaust is solved in the process of petroleum pollution to environment. However, this method has some defects which is not suitable for the mixed gas with low $\mathrm{H} 2 \mathrm{~S}$ concentration.

Iron oxide desulfurization. Iron oxide desulfurization method is the activity of iron oxide for effective removal of $\mathrm{H} 2 \mathrm{~S}$ gas, the operating conditions required to operate strictly, in alkaline conditions, and it needs to reach the $\mathrm{pH}$ by adding soda ash to realize this purpose. Due to its easy operation, simple process and low energy consumption, it is widely used in the removal of H2S gas from natural gas processing and city gas. The disadvantage is that the desulfurization agent will reduce the activity that to be regularly replaced, and covers a larger area, so the overall economic efficiency is uneconomical.

Composite zinc desulfurization. In the treatment of low concentration of $\mathrm{H} 2 \mathrm{~S}$ gas, Zinc Oxide can be used as a desulfurization agent, so that it reacts with $\mathrm{H} 2 \mathrm{~S}$ to generate a stable nature of the $\mathrm{ZnS}$ to achieve the purpose of fine desulfurization. The method has a high desulfurization effect, but because of the poor regeneration ability and slow chemical kinetics of Zinc Oxide, it is easy to cause the Zinc Oxide to be reduced to zinc, which causes the gasification of zinc at high temperature. According to the defects of Zinc Oxide, a new type of composite metal desulfurizer was developed.

Wet oxidation method. At present, the method of oxidation mainly includes the following methods: (1) the absorption of ferric oxide suspension; (2) the organic catalyst absorption oxidation method; (3) arsenic alkali method.

Absorption of ferric oxide suspension. The process of absorption of $\mathrm{H} 2 \mathrm{~S}$ in the iron oxide suspension, and the basic is the sodium carbonate or ammonia which can react with $\mathrm{H} 2 \mathrm{~S}$, namely hydrated ferric oxide will react with $\mathrm{H} 2 \mathrm{~S}$ into the fan using oxygen as oxidant, the reaction of iron sulfide and oxygen and iron oxide and sulfur. The absorption of ferric oxide suspension has a higher $\mathrm{H} 2 \mathrm{~S}$ removal efficiency, up to $85 \% \sim 99 \%$. Compared with the dry oxidation method, the construction area of the device is greatly reduced. The disadvantage is that the solution is acid and 
alkali solution which will cause some corrosion to the equipment. In order to ensure the safety of the equipment, the equipment with high material quality must be selected.

Absorption oxidation of organic catalysts. This method is the need for a reduction in catalytic oxidation method with selecting phenolic cmpounds, and adding some water to prepare aqueous solution of salts, or it can select the alkaline solution with oxygen carrier, these two kinds of organic compounds in transition from oxidation to reduction of organic compounds, reducing $\mathrm{H} 2 \mathrm{~S}$ monomer into sulphur. The absorption and oxidation process of organic catalyst has the following advantages: 1) no pollution of the effluent; 2) non-toxic absorbent solution; (3) high quality by-product sulfur; 4) higher purification efficiency.

Arsenic alkali method. Arsenic alkali method is also called Thylox, containing arsenic in alkaline solution which can effectively remove $\mathrm{H} 2 \mathrm{~S}$ gas mixture, in the desulfurization type, wet oxidation method of harmful gas sulfur removal can be used for various petroleum processing raw gas. Arsenic alkali method is widely used, but it is a highly toxic substance which can cause serious pollution to the environment. With the improvement of the requirements of the national emission standards, the method is far away from the industrial production, and it has been replaced by some other methods.

Absorption method. In the method of removing $\mathrm{H} 2 \mathrm{~S}$, it mainly includes chemical absorption method and physical absorption method.

Chemical absorption method. The chemical absorption method for removal of H2S in which the reaction solution $\mathrm{pH}$ value is controlled in $9 \sim 11$ between the weak acid and alkali salt solution, and alkaline solution does not need to be too strong. It includes the following 3 types: (1) ethanol amine absorption method; (2) sodium carbonate absorption method; (3) ammonia absorption method.

Ethanolamine method. In ethanolamine absorption method, the principle of using ethanolamine desorption under low temperature absorption and high temperature, the ethanolamine absorption of $\mathrm{H} 2 \mathrm{~S}$ gas in raw gas at low temperature, and the reaction production with salts using high temperature environment $\mathrm{H} 2 \mathrm{~S}$ gas analysis from ethanolamine. The commonly used ethanolamine includes ethanolamine and two ethanolamine. Ethanolamine method has the advantages of strong solution reaction, low price, stable chemical property and easy recovery. The disadvantage is that the solution has a serious loss of the solution and requires higher vapor pressure.

Sodium carbonate absorption method. Sodium carbonate absorption absorption solution is alkaline salt solution to absorb acid $\mathrm{H} 2 \mathrm{~S}$ gas using acid-base reaction principle, due to weak acid buffer effects, with the absorption of $\mathrm{H} 2 \mathrm{~S}$ gas, the $\mathrm{pH}$ value of the solution will not change greatly at the beginning, and it can guarantee the stability of the system in production. The sodium carbonate absorption method has the advantages of simple equipment and high efficiency, and the disadvantage is that the sodium carbonate is produced in the reaction, so as to reduce the absorption effect of $\mathrm{H} 2 \mathrm{~S}$ gas.

Ammonia absorption method. Ammonia absorption method using the absorption of H2S gas medium is alkaline solution, the utility model has the advantages of low cost desulfurizer and simple equipment, which is widely used in the coking plant and ammonia plant, on the other hand, it will be subject to certain limitations.

Physical absorption method. In the process of removing H2S gas by physical absorption method, the organic solvent is selected as the absorption solvent. Compared with the chemical absorption method, the reaction condition is required to be reduced. The disadvantage is that the concentration of H2S gas is higher, and the absorption effect is better when the concentration is high. Common physical absorption methods are: (1) cold formaldehyde method; (2) propylene carbonate; (3) -Nmethyl pyrrolidone method.

Adsorption method. At present, the main methods include: (1) activated carbon adsorption; (2) molecular sieve adsorption method.

Activated carbon adsorption method. Activated carbon adsorption method is for the desulfurization agent with solid activated carbon, the desulfurization agent at room temperature can accelerate the oxidation rate of $\mathrm{H} 2 \mathrm{~S}$, so that the production of sulfur oxidation activated carbon 
adsorption [3]. The method has the following advantages: 1) the operating temperature is low; and the process is simple; 3) the removal effect is good; and the production cost is low; 5) the H2S gas with low concentration can be removed by. Because of its advantages, it is widely used in petroleum processing and light industry.

Molecular sieve adsorption method. The molecular sieve adsorption method means due to highly concentrated charge and extremely large surface area, the concentration of charge to molecular sieve adsorption occurs strongly on $\mathrm{H} 2 \mathrm{~S}$ molecular sieve adsorption method which can not only remove $\mathrm{H} 2 \mathrm{~S}$ gas in raw gas, and it can effectively remove mercaptan, CS2 and other sulfur-containing compounds.

\section{Conclusion}

With the improvement of living standards, China's environmental haze is serious, and our country puts forward higher requirements of national emission standards on enterprise exhaust, because there are a lot of harmful gas hydrogen sulfide oil processing in raw materials, and it will cause irreversible damage to the environment directly discharged. Therefore, this article introduces a method for each $\mathrm{H} 2 \mathrm{~S}$ removal by wet oxidation method and dry oxidation method from principle, advantages and disadvantages and their process of removal of hydrogen sulfide, and it provides a theoretical basis for the process after removal of hydrogen sulfide in petroleum processing.

\section{References}

[1] Xiaoyao Tan, Diyong Wu, Quan Yuan.Reaction kinetics of H2S removal by impregnated activated carbon[J].Chemical Reaction Engineering and Technology, 1996, 12(2): 129 137.

[2] Kaixi Li,Licheng Ling.Study on adsorption and transformation of SO2 on spherical activated carbon[J].Coal Conversion, 1999, 1(22): 76 78.

[3] Jianzhi $\mathrm{Wu}, \mathrm{Chunhu} \mathrm{Li}$, Shulan $\mathrm{Yu}$ and so on.Research progress of carbon based materials for removing H2S[J].Coal Conversion,2006, 4(29): 26〜29. 